\title{
A Biomechanical Analysis System to Evaluate Physical Usability of Kimchi Refrigerator
}

\author{
Inseok Lee ${ }^{1}$, Jae Hee Park ${ }^{1}$, Tae-Joo Park ${ }^{1}$, and Jae Hyun Choi ${ }^{2}$ \\ ${ }^{1}$ Department of Safety Engineering, Hankyong National University, Anseong, 456-749, \\ South Korea \\ ${ }^{2}$ U2 Systems, Anyang, 431-070, South Korea \\ \{lis, maro\}@hknu.ac.kr, taejoo80@hanmail.net, choi2000@u2system.co.kr
}

\begin{abstract}
A biomechanical analysis system, consisting of measurement and analysis subsystems, were developed and applied in evaluating the physical usability of a kimchi refrigerator. In the system, 3D motion measurement system and force platform system were used in measuring joint positions, ground reaction forces and moments. The systems also includes 3 analysis modules: kinematic, kinetic, and 3DSSPP analyses. Kimchi refrigerator, which is very popular as a specific refrigerator for kimchi, a Korean traditional dish, was evaluated using the system. The refrigerator is designed as a top-cover that makes the users feel uncomfortable in using it, though most people think it is a very useful product. The result showed it is possible to evaluate the physical usability of the refrigerator using the system effectively and reliably.
\end{abstract}

Keywords: biomechanical analysis, 3D motion analysis, physical usability, kimchi refrigerator.

\section{Introduction}

In applied ergonomics area, biomechanical approaches have been mainly used in evaluating physical tasks like manual materials handlings [1]. In the process of product design, it seems that ergonomics is emphasized as the principle to improve the usability of a product, which is thought to be less related to biomechanical approaches. Although usability is often thought to be cognitive measures rather than physical aspects in using a product, there should be no barriers in describing and measuring how a product is easy to use. Physical usability is used in this study to specify the usability related to the physical ways of using a product.

Biomechanics is a multidisciplinary activity to investigate and describe the motions and forces of body parts during several activities [1]. When a user uses a product like a refrigerator, his/her motions and related forces on the body could be measured and analyzed through biomechanical approaches. The analysis can be connected to the physical usability of the product.

To carry out those studies, there should be some equipment to measure positions of body parts and forces related to the motion. In addition, it needs properly designed biomechanical analysis modules in which some measures, such as position, force, 
moment, velocity and acceleration, should be calculated. The system including measurement system and analysis modules can be used in evaluating physical usability of products.

The objective of this study is to develop a biomechanical analysis system using measurement systems in order to evaluate physical usability of consumer products. The main scope is to develop biomechanical analysis modules using the data obtained from the measurement system. A case study was carried out to investigate how the system works by evaluating the physical usability of a kimchi refrigerator.

\section{Biomechanical Analysis System}

The biomechanical analysis system consists of a measurement system and analysis modules. Fig. 1 shows the structure of the system and the outputs to be obtained through the analysis modules.

\subsection{Measurement System}

The system consists of 3 subsystems: a 3D motion measurement system, a ground reaction force and moment measurement system, and a digital recorder. The 3D motion measurement system is an optoelectronic motion measurement system with 5 infrared cameras (ProReflex, Qualisys, Sweden). Reflective markers are used in measuring $3 \mathrm{D}$ positions. A force platform is installed to the system to measure the ground reaction forces and moments in three directions (AMTI, USA). Finally, a digital video recorder is used to record the motions. The three subsystems are all connected together so that the measurement could be synchronized automatically.

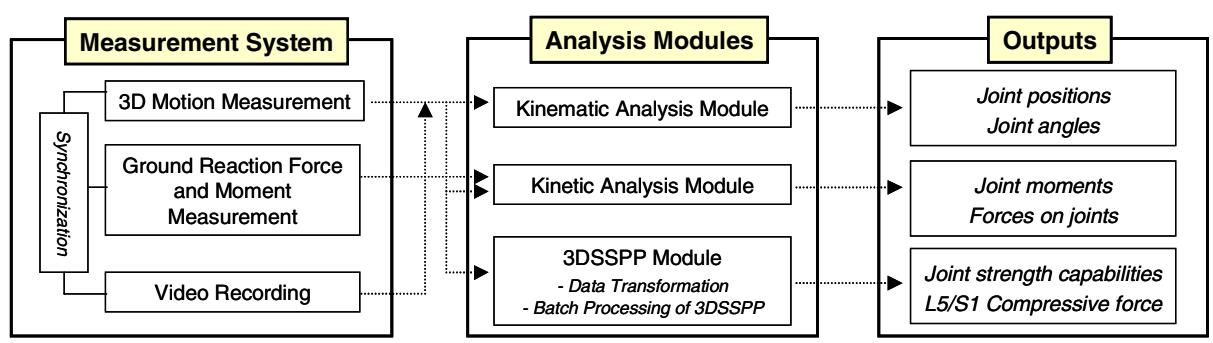

Fig. 1. Structure of the biomechanical analysis system

\subsection{Analysis Modules}

There 3 analysis modules in the system: kinematic analysis, kinetic analysis, and 3DSSPP analysis modules. In kinematic analysis module, the body motion can be analyzed by calculating joint positions, joint angles, body parts' velocities and accelerations. In this module, joint position data measured through the 3D motion measurement system are mainly used and the video recording is used in visually comparing the analyzed data. 
In the kinetic analysis module, the physical load on joints can be analyzed by calculating joint reaction forces and moments. In this module, the data from the 3D motion measurement system and the ground reaction force and moment system are mainly used. The module, developed using Microsoft Excel, includes an algorithm to find out the motion with high moments on each joint.

In the 3DSSPP analysis module, 3DSSPP (3D Static Strength Prediction Program) is used in analyzing biomechanical load on the body [2]. The program was designed to evaluate manual materials handling tasks through biomechanical analyses. In this module, an algorithm to transform the joint position data measured by the 3D motion measurement system into an input format of 3DSSPP. The transformed data is used in making a batch file to be processed by 3DSSPP for analyzing the physical load of the tasks. It is possible to analyze many motions using 3DSSPP at a time in this module. The results include the strength capabilities and compressive force at L5/S1. The module was developed using SAS and Microsofrt Excel.

\section{Case Study: Evaluation of Kimchi Refrigerator}

\subsection{Kimchi Refrigerator}

Kimchi refrigerator is a specific refrigerator for kimchi, which is a traditional Korean dish of fermented vegetables seasoned with chili peppers and salt. The kimchi is so popular in Korea that most Koreans eat it everyday and keep some quantity of kimchi at their home. Because kimchi is a fermented dish, the length of keeping its proper taste is strongly affected by the temperature and the storing way. In tradition, Koreans have stored lots of kimchi in ceramic jars buried under the ground to secure its optimal taste during winter season. However, the Korean modern housing circumstances have made it difficult for the people to take the traditional way of storing. As the society developed economically much, the people began to feel that the general refrigerator has a limit in storing lots of kimchi for a long time. The kimchi refrigerator was developed based on the social necessities about 10 years ago, and now it came to be a popular consumer product like the general refrigerator, washing machine or TV set. It is presumed that more than $80 \%$ of Korean families have their own separate refrigerator that is designed to keep kimchi at an optimal temperature for proper fermentation [3].

In a survey to investigate the customer satisfaction of kimchi refrigerator, it was shown that the most users are satisfied with the use of the refrigerator [4]. However, they also reported high discomfort related to the way of using the refrigerator, in particular, bending his/her torso very much to put kimchi cases into the refrigerator, take them out of it, or clean its inside. Seventy-five percent of the respondents reported the experience of body-part pains and 58.4\% of them reported pain experience in the back. It is presumed that these results are highly correlated to the top-cover design of the refrigerator, which is the popular style to improve its performance of maintaining the temperature properly (Fig. 2). 

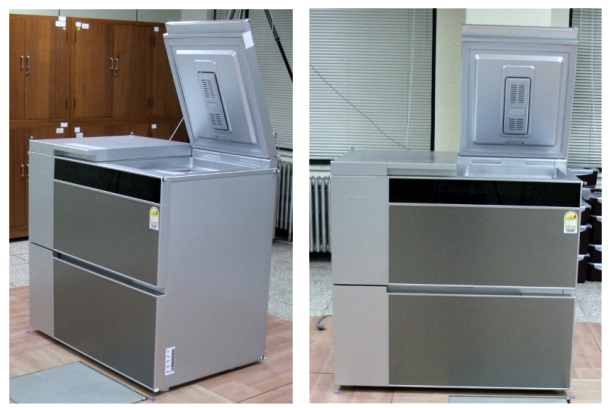

Fig. 2. An example of a popular top-cover kimchi refrigerator

\subsection{Experiment}

Subjects. Three female volunteers participated in the laboratory experiment. They have no history of any musculoskeletal diseases. They all have the experience of using kimchi refrigerator.

Measurement of Joint Positions. An optoelectronic motion measurement system with 5 infrared cameras (ProReflex, Qualisys, Sweden) was used to measure the position of joints. Reflective markers were put on 22 landmark positions: right and left sides of the head, the 7th cervical vertebra, sternum, right and left acromions, right and left elbows, right and left wrists, right and left hands, right and left pelvises, right and left hip joints, right and left knee, right and left ankle joint (Fig. 3).
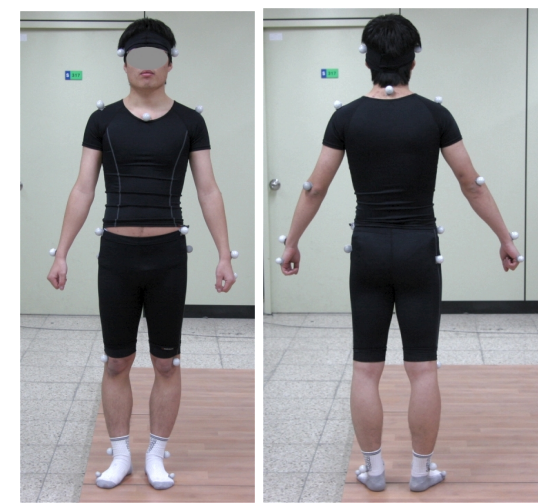

Fig. 3. The positions of markers for 3D motion analysis

Kimchi refrigerator. A kimchi refrigerator commercially manufactured was selected in the experiment (Fig 2). It has three bays to put kimchi cases or vegetables. Usually, the upper bays with top-cover are used in storing kimchi, which is kept in the specific cases. The refrigerator was designed to put 6 cases into the upper bays, which are stacked in 3 layers and 2 columns: case 1: an upper layer and forward column (close to the user); case 2: the upper layer and backward column (far from the user); case 3: 
a middle layer and forward column; case 4: the middle and backward column; case 5: a lower layer and forward column; and case 6: the lower layer and backward column.

Experimental task. The subject was asked to stand on the force platform, which is located in front of the refrigerator (Fig. 3). The subject then took out the kimchi cases, which were filled with sand and weighed $10 \mathrm{~kg}$, from the refrigerator in the order of the case numbers (1 to 6). She took out the kimchi case and stacked them on the floor by the refrigerator.
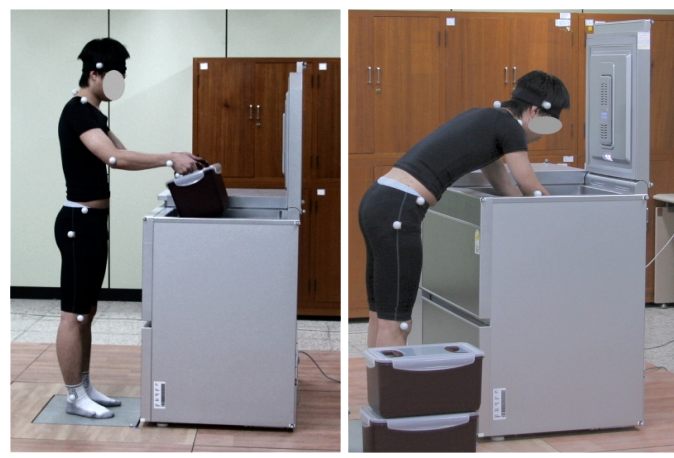

Fig. 4. The postures adopted for putting a case into the refrigerator

\subsection{DSSPP Analysis}

Using the 3DSSP analysis module, the 3D position data were transformed automatically and the batch processes of 3DSSPP were carried out. It was possible to calculate L5/S1 compressive forces at all motions of lifting the kimchi cases out of the refrigerator. As expected, the compressive forces were highest when the subject lifts up the case from the stack. The compressive forces at that moment were extracted and Fig. 5 shows the mean values. The graph shows that the compressive force increase as the layer of case is lower and the case is far away from the user. The

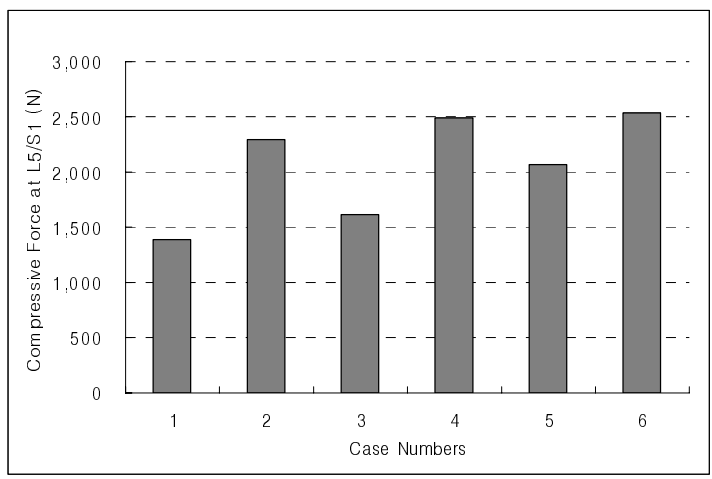

Fig. 5. Mean compressive forces at L5/S1 according to the cases to be lifted 
highest compressive force was about 2,500 $\mathrm{N}$ when lifting the case in the lower layer and backward column. Because NIOSH defined 3,400 N as the action limit [1], it can be said that the task of lifting kimchi case does not involve high risk of low back pain. However, it should be remarked that the users of the refrigerator are generally female and elderly people, and the task could involve a high risk for those relatively low capabilities.

\section{Conclusion}

In this study, a biomechanical analysis system was developed using a 3D motion and ground force measurement system. The system includes the modules to carry out kinematic, kinetic, and 3DSSPP analysis. The case to evaluate the kimchi refrigerator showed that the system could be used in evaluating the physical usability of various products effectively and reliably. In the future, more effort is necessary to make the system easy to use, and standardize and validate the methods of evaluating physical usability.

\section{References}

1. Chaffin, D.B., Andersson, G.B.J., Marin, B.J.: Occupational Ergonomics, 3rd edn. John Wiley and Sons,Inc.New York (1999)

2. University of Michigan, http://www.engin.umich.edu/dept/ioe/3DSSPP/

3. Segye Times: About the evolution of Kimchi refrigerato (in Korean) (2006.9.4)

4. Lee, I., Park, J.H., Park, T.J.: Survey of customer satisfaction of Kimchi refrigerator in the aspect of physical usability. IE Interfaces. Submitted (in Korean) 